\title{
PERSISTENT MEDIAN ARTERY ASSOCIATED WITH SUPERFICIAL RADIAL ARTERY AT THE WRIST- A CASE REPORT
}

Jayasabarinathan. M.

1. Assistant Professor, Department of Anatomy, Sri Venkateshwara Medical college and Research Centre, Ariyur, Puducherry.

\section{CORRESPONDING AUTHOR}

Dr. Jayasabarinathan. M

6/10, Veerappa Street,

Anandha nagar, Kathirkamam,

Puducherry-605009

E-mail: drjayasabarinathan@gmail.com

Ph: 00919994248438

ABSTRACT: Vascular variations are usually the result of developmental anomaly during the formation of blood vessels in any respective part of the body. In this case a rare unilateral variation was found on the left upper limb during routine dissection in the Department of Anatomy. Brachial artery divided into radial artery and a common trunk. The common trunk in turn divided into ulnar, median and common interosseous artery. The median artery was palmar type, had two proper palmar digital branches in the palm. The radial artery had normal course in the forearm till the junction between upper two third and lower one third where it turned dorsally and reached the anatomical snuff box and ran superficial to the abductor pollicis longus, extensor carpi radialis brevis and extensor carpi radialis longus along the cephalic vein medially and superficial branch of radial nerve laterally. The median artery, arose from the common trunk, had pierced the median nerve about $4 \mathrm{~cm}$ from its origin then ran along the median nerve in the forearm thereby reached the palm where it gave off two common palmar digital branches but did not anastomose with the ulnar artery hence formed incomplete superficial palmar arch. But on the right side no such variations found. These vascular variations have been studied in detail and their clinical implications and embryological significance are emphasized.

KEY WORDS: median artery, superficial radial artery, superficial palmar arch, variations

INTRODUCTION: Brachial artery is a continuation of the axillary artery and divides into radial and ulnar arteries at the level of neck of radius in the cubital fossa. The radial artery reaches the wrist where it is palpable between flexor carpi radialis and the anterior border of the radius. After passing deep to the abductor pollicis longus and extensor carpi radialis brevis the radial artery reaches the anatomical snuff box where its become a content of it. Further, it enters into the palm by piercing the first dorsal interosseous muscle. Thence it forms deep palmar arch in the palm after giving off the princeps pollicis and radialis indicis artery. A common interosseous artery arises from the ulnar artery, divides into anterior and posterior interosseous arteries. In the palm, the ulnar artery forms superficial palmar arch and it is completed laterally by superficial branch of radial artery. Ulnar artery gives off one proper palmar digital branch and three common palmar digital branches ${ }^{1}$.

This case report discusses embryological reasons and clinical significance of unilateral persistent median artery associated with superficial radial artery at the wrist. 
CASE REPORT: The present case demonstrates the variation in the branching pattern of brachial artery, course of radial artery and the formation of superficial palmar arch in the left upper extremity of a 60 yrs old male cadaver during routine undergraduate dissection in the department of Anatomy. Brachial artery divided into two branches, radial artery and a common trunk at the level neck of radius. In which, the common trunk further divided into three branches, the ulnar, median and common interosseous artery (fig.1). The ulnar artery had a normal course in the forearm but in the hand it gave off two common palmar digital branches and supplied medial two and half fingers (fig.3). The radial artery had normal course in the forearm till the junction between upper two third and lower one third where it turned dorsally and reached the anatomical snuff box and ran superficial to the abductor pollicis longus, extensor carpi radialis brevis and extensor carpi radialis longus along the cephalic vein medially and superficial branch of radial nerve laterally (fig.2). The median artery, arose from the common trunk, had pierced the median nerve about $4 \mathrm{~cm}$ from its origin then ran along the median nerve in the forearm thereby reached the palm where it gave off two common palmar digital branches and supplied lateral two and half fingers but did not anastomose with the ulnar artery hence formed incomplete superficial palmar arch (fig.3). But on the right side no such variations found.

DISCUSSION: Variations in the vascular patterns are usually the result of developmental anomaly during the formation of blood vessels in any part of the body. Udyavar observed the brachial artery dividing into radial and ulnar arteries with common interosseous artery arising from the radial artery ${ }^{2}$. But in the present case, the brachial artery divided into radial artery and a common trunk which in turn divided into ulnar artery, median artery and common interosseous artery. Potu et al, have studied the persistence of median artery in 25 upper limbs, in which the incidence of median artery was $8 \%$, of which $4 \%$ was on the right side ${ }^{3}$. Various studies have stated that the persistence of the median artery is either palmar type or antebrachial type ${ }^{4,5,6}$. Sanudo et al, found that the median artery is encircled by median nerve as in our case ${ }^{6}$. The present case showed the palmar type of median artery which did not contribute to the formation of superficial palmar arch. Coleman and Anson classified the superficial palmar arch. The present case fits into the group II type $C$ where the palm is supplied by both median and ulnar arteries but there is no anastomosis between them ${ }^{7}$.

Abdullah et al, reported a case in which radial artery had normal course till the lower third of forearm but thereafter it passed dorsally and divided into two branches superficial and deep radial arteries ${ }^{8}$. In the present case no such divisions were found. Manners-Smith classified the variants of the radial artery into 2 groups. The type I variant which is more common and involves a single dorsal division and is entirely superficial as in the present case. Type II, which is very rare and involves the dorsal division further dividing into 2 branches, superficial and deep 9 .

EMBRYOLOGICAL EXPLANATION: Arey stated that the anomalies of blood vessels may be due to: (i) Incomplete development (ii) The persistence of vessels normally obliterated (iii) The disappearance of vessels normally retained (iv) The choice of unusual paths in the primitive vascular plexuses. (v) Fusion and absorption of the parts usually distinct ${ }^{10}$.

According to Senior (1926), originally the subclavian artery extends to the wrist, where it terminates by dividing into terminal branches for the fingers. The distal portion of the artery becomes the interosseous artery of the adult. The median artery arises from the interosseous 
artery and becomes larger while interosseous artery subsequently undergoes retrogression. During this process the median artery fuses with the lower portion of interosseous artery and ultimately forms the main channel for the digital branches becoming the principle artery of the forearm. The ulnar artery arises from brachial artery and unites distally with the median artery to form superficial carpal arch. Digital branches arise from this arch. The superficial brachial artery develops in the axillary region and traverses the medial surface of the arm and runs diagonally from the ulnar to the radial side of the forearm to the posterior surface of the wrist. There it divides over the carpus into branches for the dorsum of the thumb and index finger. Finally three changes occur. The median artery undergoes retrogression becoming a small slender structure, now known as arteria comes nervi mediani. The superficial brachial artery gives off a distal branch which anastomoses with the superficial palmar arch already present. At the elbow an anastomotic branch between brachial artery and superficial brachial artery becomes enlarged sufficiently to form with the distal portion of the latter, the radial artery, as a major artery of the forearm; the proximal portion of the superficial brachial artery atrophies correspondingly ${ }^{11}$.

Normally the median artery retrogresses and the superficial palmar arch is contributed by ulnar artery but in the present case median artery persisted and it gave off two common palmar digital arteries for lateral two and half fingers. It did not anastomose with ulnar artery, resulted in development of incomplete superficial palmar arch. There is no embryological explanation for superficial position of radial artery at the wrist. In primates the radial artery divides into a volar branch, which in the human is represented by a radio palmer branch, and a dorsal branch, which is represented by the normal radial artery, which further divides into superficial and deep branches in relation to the tendons in the anatomical snuff box ${ }^{8}$.

CONCLUSION: The knowledge of unusual course and branches of the vessels are important for the diagnostic, interventional and surgical procedures. The persistence of median artery may cause compression of the median nerve as it is encircled by later. The palmar type of median artery can increase the pressure on the median nerve causing carpal tunnel syndrome ${ }^{12}$. The radial artery as a superficial structure at the wrist is susceptible both to direct penetrating injury and to blunt trauma, with the potential development of both false and true aneurysms ${ }^{13}$. The existence of a superficial radial artery implies the possible absence of a normal radial pulse at the wrist and may produce problems in cannulation ${ }^{14}$. It may cause inadvertent intra-arterial drug administration due to its proximity to the cephalic vein. So anaesthetists, surgeons and radiologists should be aware of these arterial variations to avoid unnecessary complications.

\section{REFERENCES:}

1. Susan Standring, Borley NR, Collins P, Crossman AR. Axilla. Gray's Anatomy. 40th ed. Elsevier, Spain 2008:814-817.

2. Udyavar A. Anomalous termination of the brachial artery. J Anat Soc India 2004; 53:41.

3. Potu BK, Ray B, Pai SR. A preliminary survey of the median artery in human cadavers of south Indian origin. Bratisl Lek Listy. 2011; 112(5): 292-295.

4. Rodriguez-Niedenfuhr M, Sanudo JR, Vazquez T. Median artery revisited. J Anat. 1999; $195:$ : $7-63$

5. Rohini KR, Dakshayani K. Unilateral Persistence of Median Artery and its Clinical Significance. Anatomica Karnataka. 2011; 5(2):39-42. 
6. Sanudo JR, Chikwe J, Evans SE. Anomalous median nerve associated with persistent median artery. Anat.1994;185:447-451

7. Coleman SS, Anson BJ. Arterial patterns in the hand based upon a study of 650 specimens ext. Surg Gynecol Obstet, 1961; 113(4):409-424.

8. Abdullah M. Aldahmash, Walid A. Zaher, Hasem H. Darwish. An atypical superficial radial artery Eur J Anat. 2010; 14 (1): 39-41

9. Wood SJ, Abrahams PH. Bilateral superficial radial artery at the wrist associated with a radial origin of a unilateral median artery. J Anat. $1997 ; 189: 691-693$.

10. Arey. Developmental anatomy. 6th ed. Philadelphia: W.B Saunders Co.; 1957.pp375-377.

11. Senior HD. A note on the development of the radial artery .Anat Rec. 1926; 32:220-221

12. Toranto IR. Aneurysm of the median artery causing recurrent carpal tunnel syndrome and anatomic review. Plast Reconstr Surg.1989; 84:510-512

13. Gretchen A. W. Gooding. Sonography of the Radial Artery at the Wrist. Am J Roentgenol. 1988; 150: 629-631.

14. Diz JC, Ares X, Tarrazo AM, Alvarez J, Meanos ER. Bilateral superficial radial artery at the wrist. Acta Anaes Scand, 1998; 42: 1020.

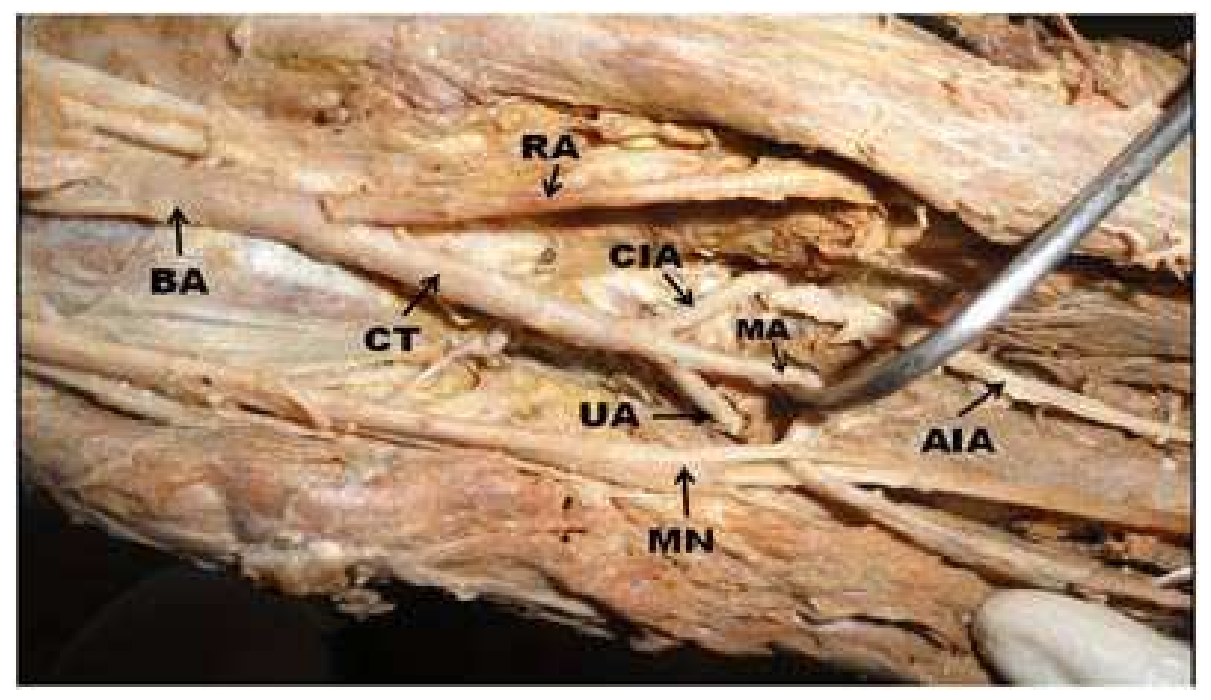

Fig.1. Showing variant branching pattern of brachial artery and persistent median artery in the left cubital fossa.
BA- Brachial Artery
CT- Common Trunk
RA- Radial Artery
UA- Ulnar Artery
MA- Median Artery
CIA- Common Interosseous Artery
AIA- Anterior Interosseous Artery
MN- Median Nerve 


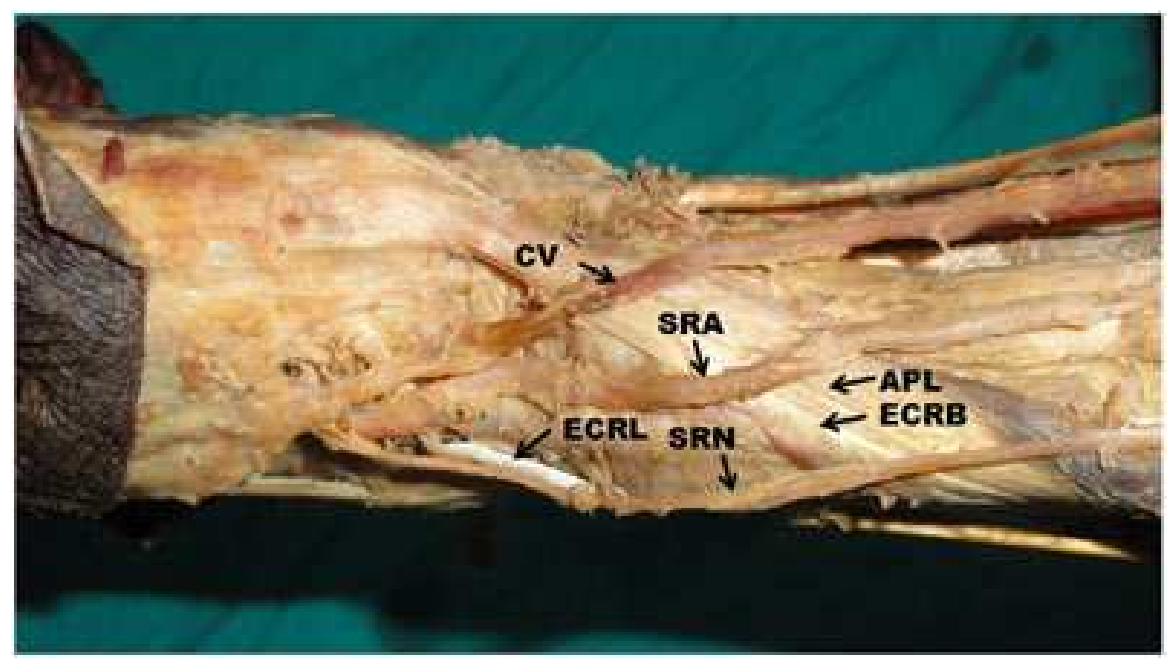

Fig.2. Showing superficial radial artery at the left wrist.
CV - Cephalic vein
SRA - Superficial Radial Artery
SRN - Superficial Radial Nerve
APL - Abductor Pollicis Longus
ECRL - Extensor Carpi Radialis Longus
ECRB - Extensor Carpi Radialis Longus

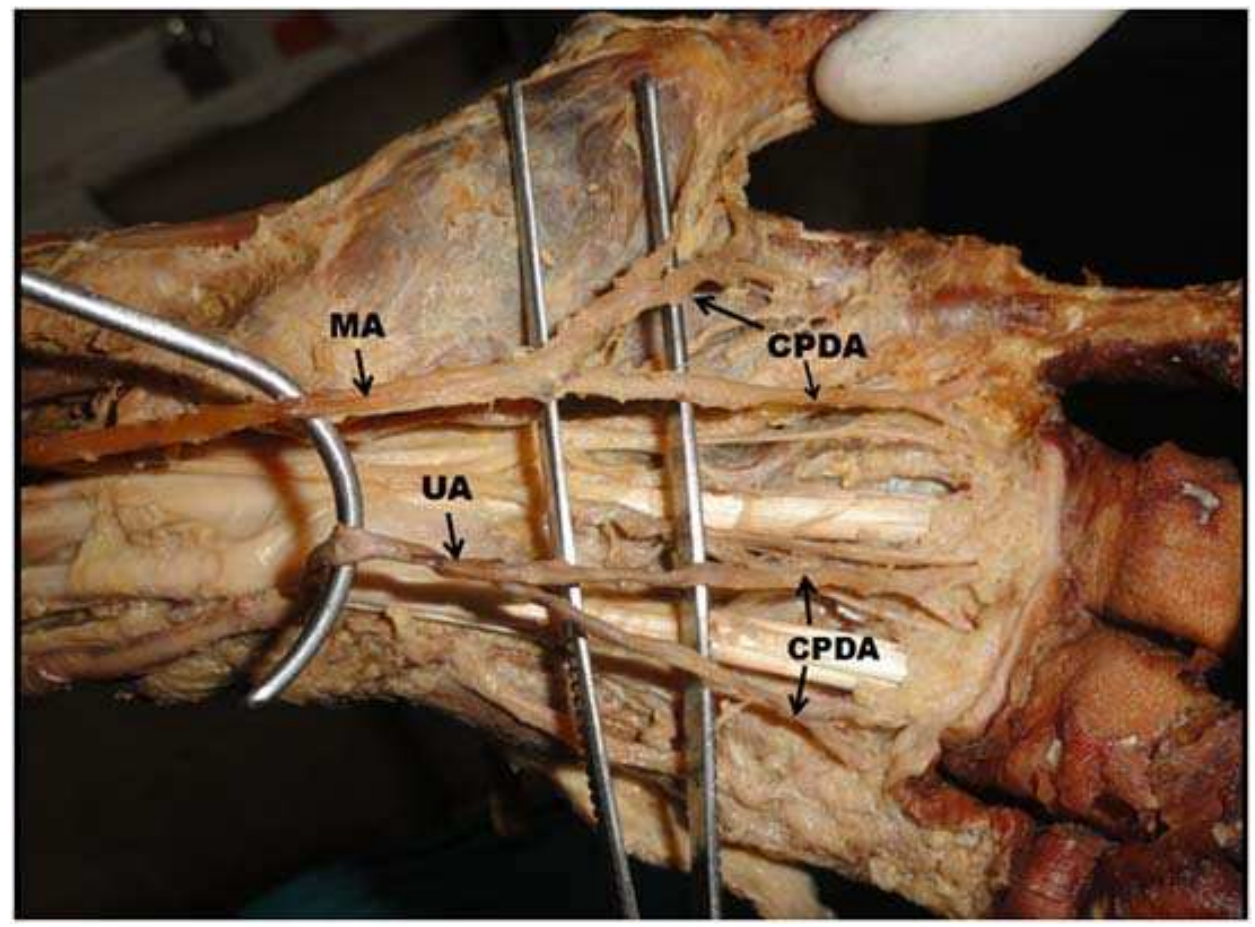

Fig.3. Showing incomplete superficial palmar arch.
MA - Median Artery
UA - Ulnar Artery
CPDA - Common Palmar Digital Artery 